\title{
Concentrations of interleukin-2 in the nasopharyngeal secretion of children with acute respiratory syncytial virus bronchiolitis
}

\author{
Katia M. Giugno, ${ }^{1}$ Denise C. Machado, ${ }^{2}$ Sérgio L. Amantéa, ${ }^{3}$ \\ Sérgio S. Menna Barreto 4
}

\begin{abstract}
Objective: To assess interleukin- 2 concentrations in nasopharyngeal secretion of children (0-24 months) with acute respiratory syncytial virus bronchiolitis, within the first 12 hours of hospital admission, and compare the levels of IL-2 with the severity of the illness.

Methods: Prospective study performed between June and August 1999. The study included 62 patients, previously healthy, hospitalized with acute viral bronchiolitis characterized by recent prodromes of coryza and/or nasal obstruction, which evolved to at least two of the following signs: respiratory dysfunction, tachypnea, wheezing or rales, and detection of respiratory syncytial virus in nasopharyngeal aspirate. The nasopharyngeal specimens were collected within 12 hours of hospital admission. The interleukin-2 levels were obtained by enzyme immunoassay. Severity of illness was assessed through oxygen saturation by pulse oximetry, Modified Clinical Score System, time of supplemental oxygen required, length of hospital stay, and mechanical ventilation. Spearman's correlation and Kruskal-Wallis test were used to compared these variables in relation to the median of interleukin-2. The chi-square test was used for categorical analysis of interleukin-2.
\end{abstract}

Results: The mean age of patients was 2.2 (1.3-4) months. Males comprised $54 \%$ of cases. Hemoglobin oxygen saturation by pulse oximetry at hospital admission was below $95 \%$ in $66.1 \%$ of patients. The mean time of supplemental oxygen use was 4.7 days $( \pm 3.54)$. The mean length of hospital stay was 4.25 days $( \pm 1.76)$. Mechanical ventilation was used in $4.8 \%$ of patients. The values of interleukin-2 in nasopharyngeal aspirates varied from 0 to $40,256 \mathrm{ng} / \mathrm{ml}$ with

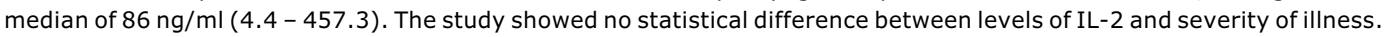

Conclusions: The interleukin-2 levels showed a heterogeneous behavior. We found no association between interleukin-2 levels in nasopharyngeal secretion and respiratory syncytial virus bronchiolitis.

J Pediatr (Rio J). 2004;80(4):315-20: Interleukin-2, acute viral bronchiolitis, respiratory syncytial virus, nasopharyngeal aspirates.

\section{Introduction}

Acute viral bronchiolitis (AVB) is the most frequent cause of hospitalization in infants younger than six months in industrialized countries. ${ }^{1}$ In our setting, AVB is responsible

1. M.Sc.; Assistant professor, Department of Pediatrics, Fundação Faculdade Federal de Ciências Médicas de Porto Alegre (FFFCMPA), Porto Alegre, RS, Brazil.

2. Ph.D.; Associate professor, Department of Internal Medicine, Pontifícia Universidade Católica do Rio Grande do Sul (PUCRS), Porto Alegre, RS, Brazil.

3. Ph.D.; Associate professor, Department of Pediatrics, Fundação Faculdade Federal de Ciências Médicas de Porto Alegre, (FFFCMPA), Porto Alegre, RS, Brazil.

4. Full professor, Department of Internal Medicine, School of Medicine, Universidade Federal do Rio Grande do Sul (UFRGS), Porto Alegre, RS, Brazil.

Manuscript received Nov 24 2003, accepted for publication May 262004. for a significant number of hospital admissions in infants younger than one year. ${ }^{2}$ The respiratory syncytial virus (RSV) is the main etiologic agent of AVB. ${ }^{3,4}$ In infants and younger children, RSV causes different levels of infection, from mild upper respiratory infection to severe lower respiratory tract infection. ${ }^{5}$ The mortality of infants hospitalized with AVB ranges from around $1 \%$ in previously healthy individuals and $3.5 \%$ in infants with a past history of cardiac or respiratory diseases. ${ }^{6}$ In our setting, results also show a low bronchiolitis-related mortality, but morbidity is significant among previously healthy infants. ${ }^{2}$ RSV infections do not provide complete immunity, so reinfections are common throughout life. ${ }^{7}$

Children infected with RSV tend to have later episodes of wheezing and asthma. ${ }^{8-10}$ The reason for this association 
is still unclear. The first hypothesis is concerned with the consequence of RSV infection, affecting the yet immature respiratory and immune systems and predisposing patients to new infections and bronchial hyperresponsiveness. ${ }^{11}$ Another hypothesis considers patient predisposition as a result of a pre-existing decrease in pulmonary function or genetic susceptibility to developing pulmonary disease. ${ }^{12}$

Information on the interactions between RSV and the human host is quite limited and the clinical behavior of RSV bronchiolitis arouses curiosity about several intriguing aspects regarding the RSV-host relationship. Clinical evidence suggests that RSV bronchiolitis is an immunomediated disease. ${ }^{3}$ The role of RSV in the pathogenesis of AVB remains obscure. ${ }^{13}$ There seems to be a delicate balance between immunopathology and immunoprotection. ${ }^{14}$ There is some evidence of the participation of $T$ cells in antiviral defense, where these cells are associated with the elimination of the virus and the development of the disease, in RSV infection. IL-2 is a cytokine responsible for the activation, growth and differentiation of $T$ cells, as well as for the induction of growth, differentiation and functional activation of a wide variety of other cells involved in the immune response. ${ }^{15}$ The IL- 2 produced by the autocrine activation of a T cell stimulates growth and the proliferation of antigen-specific T lymphocytes and B cells. ${ }^{9}$

Different results have been demonstrated in the literature regarding the behavior of IL-2, IL-4, IL-6, IL8, IL-10, IL-12, interferon-gamma, among others, measured in serum and airway secretions ${ }^{16-19}$ of patients with RSV bronchiolitis. Studies about cytokine concentrations in the nasopharyngeal secretion of patients with severe lower respiratory tract infections caused by RSV started to be published at the end of the last decade. Increase in the levels of IL-2, IL-6, IL-8, IL-10 have been reported, but some other authors could not observe an increase in the level of some of these cytokines. 16,17,20-22

More specifically, Th1 immune response, through the production of IL-2 and interferon-gamma, has aroused the interest of researchers. However, discrepant results have been found, such as those suggesting that IL-2 is an appropriate means of determining inflammatory response in patients with severe $A V B$, in the acute phase of the disease, whereas other authors could not observe Th1 immune response, as measured by interferon-gamma, at any stage of the disease. ${ }^{16,17}$

The present study assessed the role of Th1 T cells in the acute phase of the disease, determined by IL-2 production in nasopharyngeal secretion, in order to test the hypothesis that the level of this cytokine is elevated in the nasopharyngeal secretion of patients with RSV bronchiolitis, especially in the most severe cases.

The further clarification of the mechanism involved in the inflammatory response to RSV bronchiolitis may allow the development of protective measures in patients with RSV infections while viral immunization is not available.

\section{Patients and methods}

A cohort study was carried out during the peak of RSV season in Porto Alegre, between June and August 1999, including all patients aged between zero and 24 months, admitted to the Hospital da Criança Santo Antônio (HCSA) and to the pediatric intensive care unit of Hospital Moinhos de Vento (CTIP-HMV). All patients were previously healthy and had been diagnosed with AVB. The diagnosis was based on the history of prodromes with coryza and/or nasal obstruction on the last days, which led to hospital admission due to at least two of the following signs: respiratory dysfunction, tachypnea, wheezing or rales. 23 RSV was detected in the nasopharyngeal secretion of all patients by way of indirect immunofluorescence or immunoenzyme assay. Patients with history of perinatal respiratory disease, previous mechanical ventilation, previous wheezing episode, current or previous use of bronchodilators and/or corticosteroids, presence of congenital heart disease and treatment for gastroesophageal reflux or history that suggests this disease.

The nasopharyngeal secretion was obtained in the first 12 hours of hospital admission by aspiration, without instillation of physiological saline, as a part of the routine procedure for viral detection in both hospitals. A sample of $0.5 \mathrm{ml}$ was frozen in a separate flask and then kept at -80 ${ }^{\circ} \mathrm{C}$ for later IL-2 measurement. The etiologic viral diagnosis was established by indirect immunofluorescence or by immunoenzyme assay using the Abbott Testpack RSV.

All tests for IL-2 were performed in duplicates. IL-2 concentration was determined by immunoenzyme assay using the Pharmingen Human IL-2 OPT EIA Set, USA. Reading was made in a BIO-RAD Benchmark Microplate Reader at $450 \mathrm{~nm}$ and the values were obtained by linear regression analysis. The results were expressed in $\mathrm{ng} / \mathrm{ml}$. Measurements using immunoenzyme assay were made at the Laboratory of Pulmonology of the Institute of Biomedical Research of Pontifícia Universidade Católica do Rio Grande do Sul.

Researchers did not directly or indirectly indicate the hospitalization of patients. This indication could only be made by the assistant medical staff. All patients were followed up to hospital discharge, and researchers did not interfere with the treatment and/or criteria for hospital discharge.

Gender, age and weight were analyzed for characterization of the sample. The severity of AVB is assessed by measuring the hemoglobin oxygen saturation under room air conditions with an Ohmeda oximeter model 3800 (USA), at hospital admission, Modified Clinical Scoring System (Table 1), time on supplemental oxygen, length of hospital stay and necessity of mechanical ventilation (MV). ${ }^{23}$

The data were collected by three intensivists and three resident doctors (from the third year of pediatric intensive care medicine). 
Table 1 - Modified Clinical Scoring System

\begin{tabular}{lllcc}
\hline Score & $\begin{array}{l}\text { Respiratory } \\
\text { rate (RR) }\end{array}$ & Wheezing & $\begin{array}{c}\text { Saturation } \\
\mathbf{( \% )}\end{array}$ & $\begin{array}{c}\text { Use od accessory } \\
\text { muscles }\end{array}$ \\
\hline 0 & $<30$ & None & $\geq 95$ & None \\
1 & $31-45$ & $\begin{array}{l}\text { End of exhaling } \\
\text { (with stethoscope) }\end{array}$ & $90-94$ & + \\
2 & $46-60$ & $\begin{array}{l}\text { Total exhaling and inhaling } \\
\text { (with stethoscope) }\end{array}$ & $<90$ & ++ \\
3 & $>60$ & $\begin{array}{l}\text { Exhaling and inhaling } \\
\text { (without stethoscope) }\end{array}$ & $<85$ & +++ \\
\hline
\end{tabular}

Score values and classification of severity: $<3$ normal, 4-6 mild, 7-9 moderate, 10-12 severe.

Modified from De Boeck et al. ${ }^{23}$

\section{Statistical analysis}

The categorical variables were described by way of proportions. Continuous variables with normal distribution were described as means and standard deviation. IL-2 was described using median and interquartile range (25-75th percentiles).

Severity variables were categorized as follows: oxygen saturation ( $\geq 95 \%, 90$ to $94 \%,<90 \%$ ), Modified Clinical Scoring System ( $<3$ normal, 4 to 6 mild, 7 to 9 moderate, 10 to 12 severe), time on supplemental oxygen (not used, 1 to 3 days, 4 to 7 days, 8 days or longer), length of hospital stay (up to 5 days, 6 to 10 days, longer than 10 days), and necessity of mechanical ventilation (yes or no). The IL-2 median values were compared with the different categories of severity variables using the nonparametric KruskalWallis test.

Spearman's bivariate correlation analysis was used to assess the association between IL-2 and the disease severity variables by a continuous form.

In addition, IL-2 was categorized into two strata according to the median and compared using the chi-square test with the outcome variables. The Mann-Whitney test was used to assess the differences in the medians of severity variables between the two IL-2 strata.

$P$ values $\leq 0.05$ were established as significant. The Statistical Package for Social Science (SPSS) was used for the statistical analysis.

The sample size was estimated at 32 patients for differences of IL-2 between the scores (normal to severe) around $8,000 \mathrm{ng}$ and at 60 patients for differences of approximately $3,000 \mathrm{ng}$ between $\geq 95 \%$ and $<95 \%$ oxygen saturation, considering an alpha of 0.05 and a beta of 0.20 . An $n$ of 62 patients was estimated to detect a correlation of 0.35 with an alpha of 0.05 and a beta of 0.20 .
The study protocol was approved by the Research Ethics Committee of the HCSA (Santa Casa Hospital Complex) and approved by the heads of the services involved. An informed consent was signed by parents or legal guardians.

\section{Results}

Ninety-six patients with AVB were admitted to the HCSA during the study period. RSV was found in the nasopharyngeal secretion of $51(53.1 \%)$ patients. Two infants were excluded for they were using corticosteroids at admission; so the sample consisted of 49 patients. Twenty-four patients with AVB were admitted to the CTIP-HMV. Of these patients, 15 $(62.5 \%)$ had RSV in their nasopharyngeal secretion. Two patients were lost to follow-up, so the sample consisted of 13 patients.

The study included 62 infants. The medians of age and weight distribution were respectively $2.2(1.3-4)$ months and $5050(3,800-6,675) \mathrm{g}$. Thirty-four patients $(54 \%)$ were male.

The results of IL-2 concentrations showed a great variation. The IL-2 values for the nasopharyngeal secretion ranged from 0 to $40,256 \mathrm{ng} / \mathrm{ml}$ with a median of $86 \mathrm{ng} / \mathrm{ml}$ $(4.4-457.3)$.

The assessment of the severity of AVB by determining hemoglobin oxygen saturation showed that arterial oxygenation was compromised in $66.1 \%$ of the patients, in whom oxygen saturation was below $95 \%$, as measured by pulse oximeter. Oxygenation was more severely compromised in $20.9 \%$ of patients, being characterized by oxygen saturation below $90 \%$. No correlation was observed between IL-2 levels in nasopharyngeal secretion and oxygen saturation ( $r=-0.01, p=0.93$ ), as well as no statistically significant differences in IL-2 levels between different oxygen saturation ranges ( $p=0.47$ ) (Table 2 ). 
Table 2 - Hemoglobin oxygen saturation in room air and concentrations of IL-2 in the nasopharyngeal secretions

\begin{tabular}{lccc}
\hline Saturation & $\mathbf{n}(\%)$ & $\begin{array}{c}\text { IL-2 } \\
\text { median (IIQ) }\end{array}$ & $\mathbf{p}$ \\
\hline$\geq 95 \%$ & $21(33.9)$ & $67.6(2.6-2664.8)$ & \\
$90-94 \%$ & $28(45.2)$ & $80.9(6.0-159.8)$ & 0.469 \\
$<90 \%$ & $13(20.9)$ & $159.9(6.4-1730.4)$ & \\
\hline
\end{tabular}

The assessment of the severity of AVB by way of the Modified Clinical Scoring System showed that $46.8 \%$ of the cases had moderate to intense disease. The disease was mild in $41.9 \%$ of the patients. Normal scores were observed in $11.3 \%$ of the patients. The comparison of IL2 levels in nasopharyngeal secretion with the different severity score values was not statistically significant ( $p=$ 0.63 ) (Table 3). No correlation was noted between these parameters when they were analyzed as continuous variables $(r=0.16, p=0.20)$.

Table 3 - Modified clinical scoring system and concentrations of IL-2 levels in nasopharyngeal secretion

\begin{tabular}{lccc}
\hline Score & $\mathbf{n}(\%)$ & $\begin{array}{c}\text { IL-2 } \\
\text { median (IIQ) }\end{array}$ & $\mathbf{p}$ \\
\hline Normal & $7(11.3)$ & $18.2(0.4-25779)$ & \\
Mild & $26(41.9)$ & $50.4(2.22-210.3)$ & 0.633 \\
Moderate & $24(38.7)$ & $125.6(9.9-446.1)$ & \\
Severe & $5(8.1)$ & $581.6(5.5-1567.9)$ & \\
\hline
\end{tabular}

The mean time on supplemental oxygen was of 4.7 days $( \pm 3.54)$. Similarly to the other severity criteria analyzed, time on supplemental oxygen did not show any statistically significant difference as compared with IL-2 levels ( $p=$ 0.42 ) (Figure 1 ). No correlation was observed between these parameters when they were analyzed as continuous variables $(r=0.09, p=0.50)$.

Mean length of hospital stay was of 4.25 days $( \pm 1.76)$ and no statistically significant difference was found between IL-2 levels and length of hospital stay $(p=0.11)$ (Figure 2), and no correlation was observed between parameters when they were analyzed as continuous variables $(r=0.06, p=0.63)$.

The necessity of mechanical ventilation occurred in $4.8 \%$ of the patients. No statistically significant difference was found in IL-2 levels between patients with or without necessity of mechanical ventilation ( $p=0.48$ ).

IL-2 categorized according to its median was assessed in relation to outcome variables, and no statistically significant differences were noted with regard to hemoglobin oxygen saturation in room air, Modified Clinical Scoring System, time on supplemental oxygen, length of

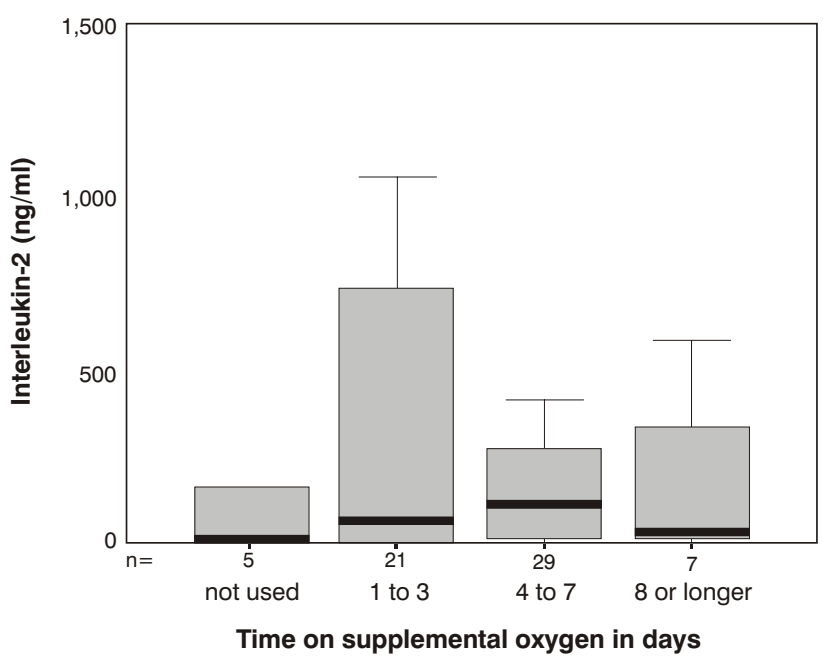

Figure 1 - IL-2 levels in the nasopharyngeal aspirate and time of use of oxygen in patients with AVB caused by RSV

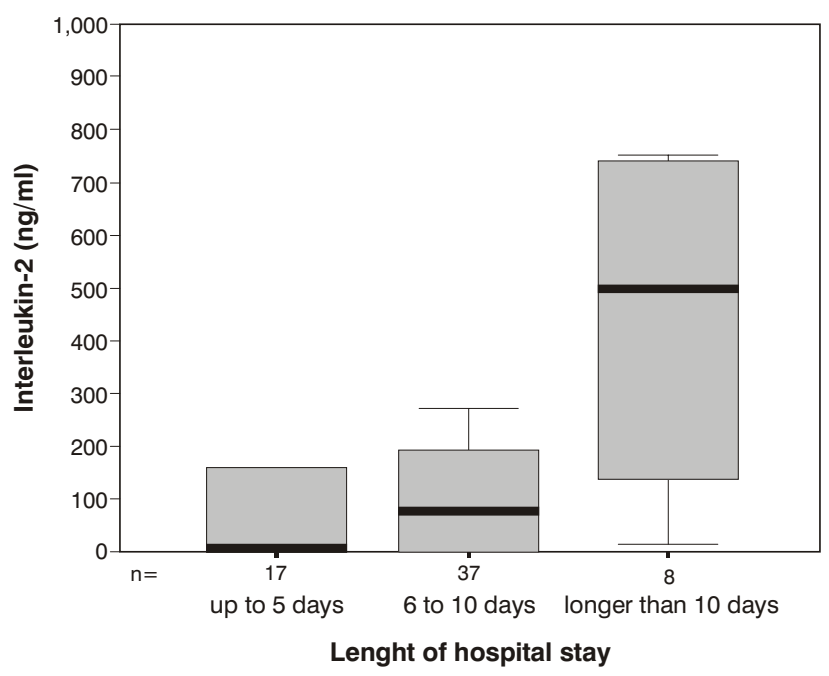

Figure 2 - IL-2 levels in the nasopharyngeal aspirate and length of hospital stay of patients with ABV caused by RSV

hospital stay and necessity of mechanical ventilation. No statistically significant differences were seen between the medians of these variables when compared in two interleukin categories.

\section{Discussion}

AVB has multiple clinical manifestations and various levels of pulmonary involvement. The rudimentary knowledge about the triggering mechanisms of AVB, which is highly prevalent in the pediatric population in all continents, has encouraged a constant search for answers.

The aim of the present study was to investigate the involvement of cellular immunity in AVB, measured through 
cytokine levels. Despite the discrepant results obtained in studies with human beings, the role of T cells (especially Th1 cells) in the elimination of the viral pathogen and in the etiology of the disease (primary or secondary infection) $)^{10}$ has been well established in animal models. The detection of cytokines in cultured peripheral blood cells from adult patients showed Th1 immune response to RSV, as measured by IL-2 and interferon-gamma levels. ${ }^{18}$ More recently, the investigation into cellular immunity as a whole includes studies on the levels of different interleukins in airway secretions. ${ }^{16,17,20-22}$ In an attempt to understand the complex role of IL-2 in immune response, we measured the levels of this cytokine in our study. Our aim was to test out the hypothesis that IL-2 was elevated in the airway secretions of patients with RSV bronchiolitis with indication for hospitalization. ${ }^{16}$

Based on the results obtained by Joshi et al., who demonstrated that IL-2 concentrations in upper and lower airways have the same behavior in AVB, we decided to determine the levels of this cytokine in nasopharyngeal secretion ${ }^{17}$. The easy collection of this material combined with the low risk of injury to the patients led us to choose this method instead of bronchoalveolar lavage, which is used to collect secretions from the lower airways.

The disease severity showed that our sample was representative of the behavior of $A V B$, as described by different authors. 24,25,27-29

No statistically significant difference was observed between IL-2 levels in nasopharyngeal secretion and disease severity assessed by hemoglobin oxygen saturation (pulse oximetry), Modified Clinical Scoring System, time on supplemental oxygen, length of hospital stay, and necessity of MV. Necessity of intensive care admission was not considered in the assessment of disease severity, since patients were selected from two hospitals with distinct healthcare characteristics.

This study was designed to detect clinically significant correlations. Although there might have been a lower statistical power for associations below the pre-established level, these values were chosen to avoid values of clinically insignificant associations. The results of our study are consistent with those reported by other authors who assessed the role of Th1 cells in different materials, both in animals and in human beings, but could not show the expected increase in cytokines associated with these cells during RSV-induced lower respiratory infections.9,16 This study suggests that IL-2 is not a good parameter to assess the immune response in RSV bronchiolitis at hospital admission if compared to the easier detection of elevated levels of other cytokines in this disease.

The medical literature shows correlations between elevated levels of interleukins in the acute phase of AVB and disease severity. Elevated IL-2 and IL- 8 levels have been found in patients submitted to MV, due to RSV bronchiolitis. ${ }^{16,17}$ Due to the small number of patients submitted to MV, the present study could not elucidate the behavior of IL-2 in the nasopharyngeal secretion of patients, exclusively with very severe AVB, defined by the necessity of MV. This can be dealt with in another study that includes several centers or more than one RSV epidemic, if we consider the percentage of patients with RSV bronchiolitis that require $\mathrm{MV}$.

Recent studies suggest that the proportion of cytokines released in inflammatory processes may be related to the differences determined by genetic polymorphisms, which should be dealt with in new studies about AVB. ${ }^{30}$

The results of this study add to the ones reported in the literature as they also attempt to clarify the pathogenesis of RSV bronchiolitis. Further investigation is necessary to improve the management of AVB.

\section{Acknowledgments}

The authors thank Helena Müller, Ângela Bagatini, Cláudia Costa e Silva, Luciano Guerra and Sônia Mastela for their help in recruiting patients for the study.

\section{References}

1. Shay DK, Holman RC, Newman RD, Liu LL, Stout JW, Anderson LJ. Bronchiolitis-associated hospitalizations among US children, 1980-1996. JAMA. 1999;282:1440-6.

2. Rubin FM, Fischer GB. Características clínicas e da saturação transcutânea de oxigênio em lactentes hospitalizados com bronquiolite viral aguda. J Pediatr (Rio J). 2003;79:435-42.

3. Kimpen JLL, Heymans HSA. Respiratory syncytial virus: immunity and immune injury. Immunol Infect Dis. 1993;3:281-8.

4. Anderson LJ, Heilman CA. Protective and disease-enhancing immune responses to respiratory syncytial virus. J Infect Dis. 1995; 171:1-7.

5. Simoes EAF, Estrany XC. Impact of severe disease caused by respiratory syncytial virus in children living in developed countries. Pediatr Infect Dis J. 2003;22:S13-20.

6. Welliver RC. Respiratory syncytial virus and other respiratory viruses. Pediatr Infect Dis J. 2003;22:S6-12.

7. Hall CB, McCarthy CA. Respiratory syncytial virus. In: Mandell GL, Bennett JE, Dolin R, editors. Mandell, Douglas, and Bennett's Principles \& Practice of Infectious Diseases. 5th ed. Philadelphia: Churchill Livingstone; 2000.

8. Sigurs N, Bjarnason R, Sigurbergsson F, Kjellman B. Respiratory syncytial virus bronchiolitis in infancy is an important risk factor for asthma and allergy at age 7. Am J Respir Crit Care Med. 2000;161:1501-7.

9. Hussell T, Spender LC, Georgiou A, O'Garra A, Openshaw PJ. Th1 and Th2 cytokine induction in pulmonary $\mathrm{T}$ cells during infection with respiratory syncytial virus. J Gen Virol. 1996;77:2447-55.

10. Kimpen JLL. Respiratory syncytial virus immunology. Pediatr Allergy Immunol. 1996;7 Suppl 9:S86-90.

11. Openshaw PJM, Dean GS, Culley FJ. Links between respiratory syncytial virus bronchiolitis and childhood asthma: clinical and research approaches. Pediatr Infect Dis J. 2003;22:S58-65.

12. Martinez F. Viruses and atopic sensitization in the first years of life. Am J Respir Crit Care Med. 2000;162:S95-9.

13. Prince GA, Horswood RL, Chanock RM. Quantitative aspects of passive immunity to respiratory syncytial virus infection in infant cotton rats. J Virol. 1985;55:517-20.

14. Ruuskanen O, Ogra PL. Respiratory syncytial virus. Curr Probl Pediatr. 1993;23:50-79.

15. Bont L, Heijnen CJ, Kavelaars A, van Aalderen WM, Brus F, Draaisma JT, et al. Monocyte IL-10 production during respiratory syncytial virus bronchiolitis is associated with recurrent wheezing in a one-year follow-up study. Am J Respir Crit Care Med. $2000 ; 161: 1518-23$. 
16. Bont $L$, Heijnen $C$ J, Kavelaars A, van Aalderen WM, Brus $F$, Draaisma JT, et al. Peripheral blood cytokine responses and disease severity in respiratory syncytial virus bronchiolitis. Eur Respir J. 1999;14:144-9.

17. Joshi P, Kakakios A, Jayasekera J, Isaacs D. A comparison of IL2 levels in nasopharyngeal ans endotracheal aspirates of babies with respiratory syncytial viral bronchiolitis. J Allergy Clin Immunol. 1998;102:618-20.

18. Jackson M, Scott R. Different patterns of cytokine introduction in cultures of respiratory syncytial (RS) virus-specific human $T_{H}$ cell lines following stimulating with RS virus and RS virus protein. J Med Virol. 1996;49:161-9.

19. Renzi PM, Turgeon JP, Yang JP, Drblik SP, Marcotte JE, Pedneault, et al. Cellular immunity is activated and a $\mathrm{TH}-2$ response is associated with early wheezing in infants after bronchiolitis. J Pediatr. 1997;130:584-93.

20. Sheeran P, Jafri H, Carubelli C, Saavedra J, Johnson C, Krisher $\mathrm{K}$, et al. Elevated cytokine concentrations in the nasopharyngeal and tracheal secretions of children with respiratory syncytial virus disease. Pediatr Infect Dis. 1999;18:115-22.

21. Abu-Harb M, Bell F, Finn A, Rao WH, Nixon L, Shale D, et al. IL8 and neutrophil elastase levels in the respiratory tract of infants with RSV bronchiolitis. Eur Respir J. 1999;14:139-43.

22. Hornsleth A, Klug B, Nir M, Johansen J, Hansen KS, Christensen LS, et al. Severity of respiratory syncytial virus disease related to type and genotype of virus and to cytokine values in nasopharyngeal secretions. Pediatr Infect Dis J. 1998;17: 1114-21.

23. De Boeck K, Van der Aa N, Van Lierde S, Corbeel L, Eeckels R. Respiratory syncytial virus bronchiolitis: a double-blind dexametasone efficacy study. J Pediatr. 1997;131:919-21.

24. Simoes EA. Respiratory syncytial virus infection. Lancet. 1999;354:847-52.
25. Behtendt CE, Decker MD, Burch DJ, Watson PH. International variation in the management of infants hospitalized with respiratory syncytial virus. Eur J Pediatr. 1998;157:215-20.

26. Wang EEL, Law BJ, Boucher FD, Stephens D, Robinson JL, Dobson S, et al. Pediatric Investigators Collaborative Network on Infections in Canada (PICNIC) prospective study of risk factors and outcomes in patients hospitalized with respiratory syncytial virus lower respiratory tract infection. J Pediatr. 1995; 126:212-9.

27. Wang EEL, Law BJ, Boucher FD, Stephens D, Robinson JL, Dobson S, et al. Pediatric Investigators Collaborative Network in Canada (PICNIC) study of admission and management variation in patients hospitalized with respiratory syncytial viral lower respiratory tract infection. J Pediatr. 1996;129:390-5.

28. Rodriguez WJ, Gruber WC, Groothuis JR, Simoes EAF, Rosas AJ, Lepow M, et al. RSV-IGIV Study Group. Respiratory syncytial virus immune globulin treatment of RSV lower respiratory tract infection in previously healthy children. Pediatrics. 1997;100: 937-42.

29. Leader S, Kohlhase K. Recent trends in severe respiratory syncytial virus (RSV) among US infants, 1997 to 2000. J Pediatr. 2003; 143:S127-32.

30. Villar J, Flores C, Méndez-Alvarez S. Genetic susceptibility to acute lung injury. Crit Care Med. 2003;31:S272-75.

Corresponding author:

Katia M. Giugno

Av. Iguaçú, 165/405

CEP 90470-430 - Porto Alegre, RS, Brazil

Tel./Fax: +55 (51) 3334.1247

E-mail: kmgiugno@terra.com.br 